\title{
Constitutional mismatch repair deficiency syndrome
}

INSERM

\section{Source}

INSERM. (1999). Orphanet: an online rare disease and orphan drug data base.

Constitutional mismatch repair deficiency syndrome. ORPHA:252202

Constitutional mismatch repair deficiency syndrome is a rare, inherited cancer-

predisposing syndrome characterized by the development of a broad spectrum of

malignancies during childhood, including mainly brain, hematological and gastrointestinal cancers, although embryonic and other tumors have also been occasionally reported. Non-neoplastic features, in particular manifestations reminiscent of neurofibromatosis type 1 (e.g., café-au-lait spots, freckling, neurofibromas), as well as premalig nant and non-malignant lesions (such as adenomas/polpyps) are frequently present before malignancy development. 